Gefässchirurgie $2022 \cdot 27: 3-4$

https://doi.org/10.1007/s00772-022-00859-4

Angenommen: 7. Januar 2022

(c) The Author(s), under exclusive licence to Springer Medizin Verlag GmbH, ein Teil von Springer Nature 2022

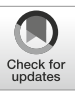

\section{Autor}
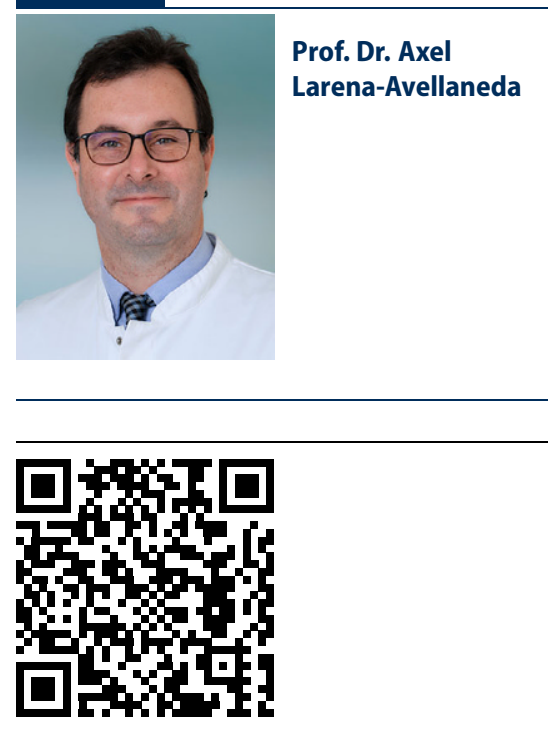

QR-Code scannen \& Beitrag online lesen

\title{
Vaskuläre Kompressionssyndrome - Chamäleons der Gefäßchirurgie
}

\author{
Axel Larena-Avellaneda \\ Abteilung für Gefäß- und endovaskuläre Chirurgie, Asklepios Klinik Altona, Hamburg, Deutschland
}

\section{Geschätzte Leserinnen und Leser,}

Herzlich willkommen in 2022! Wir starten gleich mit etwas besonderem. Vaskuläre Kompressionssyndrome sind selten, sollte man als Gefäßmediziner aber kennen und einschätzen können. Sebastian Debus hat dieses spannende Themenheft zusammengestellt. Da er bewusst die Artikel zum Dunbar-, Thoracic-outlet- und poplitealem Entrapment-Syndrom unkommentiert lässt, möchte ich die Gelegenheit nutzen, Ihnen hier meine Gedanken zu diesen, wie ich finde, nicht einfachen Krankheitsbildern darzulegen.

Susanne Honig hat einen schönen Übersichtsartikel zum Dunbar-Syndrom, in der englischen Fachliteratur meist als MALS („medium arcuate ligament syndrome") bezeichnet, verfasst. Sie schreibt: „Das Truncus-coeliacus-Kompressionssyndrom ist ein hinsichtlich Existenz, Pathogenese und Behandlung umstrittenes Krankheitsbild“. Dem kann ich nur zustimmen. Die Meinungen hierzu liegen diametral auseinander. So werben verschiedene Einrichtungen mit ihrer hohen Expertise auf dem Gebiet der Diagnostik und operativen Versorgung dieses Krankheitsbildes. Demgegenüber stehen aber auch Aussagen renommierter Gefäßchirurgen: „Dieses Krankheitsbild gibt es nicht" oder auch "So was operieren wir nicht". Tatsächlich sind die Kompressionssyndrome im klinischen Alltag oft schwer einzuschätzen, vor allem, wenn die Patienten schon mit einer vorgefassten Meinung in die Sprechstunde kommen, weil ein Arzt zufällig gesehen hat, dass die Arterie eingeengt sein könnte. Gerade hier geht es darum, die richtigen Patienten zu behandeln. Es gibt nur wenige Artikel, die den Langzeiterfolg nach Operation des Truncus-coeliacusKompressionssyndroms beschreiben. Als negativer Prädiktor für eine erfolgreiche Operation gelten u.a. psychiatrische Auffälligkeiten (!) [1]. In einer anderen Arbeit wurde über die Erfahrung von 25 Fällen mit einer Nachbeobachtungszeit von 60 Monaten berichtet [2]. Hierbei wurde bei 8 Patienten eine komplette und bei einem eine teilweise Besserung der Beschwerden angegeben. Im Umkehrschluss heißt das, 16 von 25 Patienten oder $64 \%$ hatten keine Linderung (!). Dies erklärt vielleicht auch den bösen Spruch, dass es sich bei der Operation eines DunbarSyndroms um den Gefäßeingriff mit der höchsten Mortalität handele, weil sich die Patienten hinterher selbst umbringen. Auch ich habe solche Fälle erlebt, und eine psychologische Mitbetreuung erscheint bisweilen sehr sinnvoll. Auf der anderen Seite erinnere ich mich an eine Schülerin, die wir im UKE operativ behandelten, die dann nach einem Jahr Schulausfall (!) nach der Operation wieder zur Schule gehen konnte.

\section{》) Als negativer Prädiktor für eine erfolgreiche Operation gelten psychiatrische Auffälligkeiten}

Wie so oft in der Medizin gilt es hier also, die richtigen Patienten zu finden. Untersuchungsmethode im Verdachtsfall ist der Ultraschall (s. a. Artikel von E. Stegemann). Meist sind es Frauen, und oft sind sie sehr schlank. Es verwundert dann wenig, dass in der gleichen Patientin weitere Kompressionssyndrome gefunden werden: Das Nussknacker-Syndrom (Kompression der V. renalis sinistra zwischen Aorta und A. me- 
senterica superior, siehe auch den Artikel von Thomas Scholbach in dieser Ausgabe), ggf. sogar kombiniert mit einer „pelvic congestion", und das May-Thurner(V.-iliaca-Kompressions)-Syndrom. Die Prävalenz des Beckenkompressionssyndroms wird in der Literatur mit 15-43\% angegeben (!) [3]. Bisweilen gewinnt man den Eindruck, man muss nur die richtige Krankheit für seine Methoden finden. Dies ist meines Erachtens auch der Grund, warum inzwischen viele Viszeralchirurgen das DunbarSyndrom operieren: Weil sie es können, und sie sind laparoskopisch oder gar mit dem Roboter sicher geübter als die meisten Gefäßchirurgen. Wie sagt das Sprichwort (leicht abgewandelt): „Wenn man einen neuen Hammer in der Hand hält, sieht fast alles aus wie ein Nagel". Tatsächlich ist es ja die Oberbauchanatomie der laparoskopischen Fundoplicatio, die zum täglichen Brot des Viszeralchirurgen gehört. Ob das Gefäß dann immer adäquat behandelt wird, sei dahingestellt.

Auch das Wilkie- oder A.-mesentericaKompressionssyndrom wird gerne von Viszeralchirurgen operiert. Analag zur V. renalis sinistra (Nussknacker-Syndrom) wird hier das Duodenum bei sehr schlanken Patienten mit einem steilen Abgangswinkel der A. mesenterica superior eingeengt. Operativ geht der Viszeralchirurg das mit einer Duodenojejunostomie an, - laparoskopisch aber auch gerne mit dem Roboter [4]. Dabei finde ich den Ansatz aus der Düsseldorfer Schule eleganter, die A. mesenterica superior nach kaudal zu transponieren [5]. Aber es bleibt eine Rarität, ein Wilkie-Syndrom ist noch viel seltener als ein Dunbar-Syndrom.

Aber neben dem Abdomen gibt es ja noch andere Regionen mit Kompressionssyndromen. Jeder Gefäßchirurg kennt das Thoracic-outlet-Syndrom (TOS, s. a. CMEArtikel von Thomas Bürger). Bei korrekter Indikation ist die operative Entfernung der Hals- (wenn vorhanden) und 1. Rippe sicher segensreich. Aber wie ist es, wenn Sie weder eine arterielle, venöse oder neurologische objektivierbare Pathologie finden? Operieren Sie ein „unspezifisches TOS“? Das Komplikationsspektrum dieser Operation ist nicht unerheblich, und viele $\mathrm{Pa}$ tienten sind jung und haben noch viele Erwerbsjahre vor sich. Eine Affektion des Plexus brachialis ist anatomisch bedingt sicher gar nicht selten. Und ganz ehrlich - finden Sie, die transaxilläre Resektion einer Rippe durch den kleinen Zugang ist ein sehr ästhetischer Eingriff? Ich gebe es zu - dieser gehört nicht zu meinen Lieblingseingriffen.

Viel eleganter finde ich die operative Behandlung des poplitealen Entrapments. Mit einem großen „Aber". In den meisten Fällen handelt sich es sich ja um einen funktionellen Typ 6, d.h. sie finden in der Bildgebung keine Normvariante der Gefäße. Im Operationsbericht wird dann immer das Lösen eines „bindegewebigen Strangs" oder ähnliches beschrieben. Nach wenigen Monaten kommt es oft zu einem Rezidiv. Würden Sie erneut operieren? Wie sieht es mit Alternativen aus - Muskeln einkerben, Botulinustoxin [6]? In der eigenen Praxis habe ich 2-mal sonographisch gesteuert bei einem Rezidiv Botulinustoxin injiziert. Holger Diener nennt diese Option ebenfalls am Ende seines Artikels, Langzeitdaten fehlen auch hier. Den meisten Aufwand machte bei uns das Erstellen der juristisch abgesicherten Aufklärung, da das Medikament hierfür nicht zugelassen ist. In beiden Fällen war die Therapie übrigens erfolgreich.

Gerade bei den vaskulären Kompressionssyndromen sollten wir eine strenge Indikation stellen. Ein in Exspiration sonographisch veränderter Gefäßfluss im Truncus coelicus ist häufig, ebenso ein Pulsverlust bei Anheben des Armes. Auch eine ektatisch veränderte $V$. ovarica muss keinen Krankheitswert besitzen.

\section{I) Vaskuläre Kompressionssyndro- me sollten einer strengen Indikation unterliegen}

Von daher freue ich mich über die vorliegende Ausgabe sehr. Neben den Publikationen zu den Kompressionssyndromen als Leit- und CME-Artikel finden Sie eine Übersicht zur Frage, wie Operationszeit und perioperative Komplikationen zusammenhängen (R. Grundmann), aus der Rubrik PAVK eine evidenzbasierte Broschüre für Betroffene (C. Behrendt), Teil 2 der hyperspektralen Bildgebung $(K$. Wolters für die NGG) und einen besonderen Fall, der darstellt, wie COVID-19 die Betreuung unserer Patienten negativ beeinflussen kann (L. Dienemann).
Herzliche Grüße,

$\mathrm{Ihr}$

$$
\text { Axel huena - Neblende }
$$

Prof. Dr. Axel Larena-Avellaneda

\section{Korrespondenzadresse}

Prof. Dr. Axel Larena-Avellaneda

Abteilung für Gefäß- und endovaskuläre

Chirurgie, Asklepios Klinik Altona

Paul-Ehrlich-Str. 1, 22763 Hamburg,

Deutschland

a.larena@asklepios.com

Interessenkonflikt. A. Larena-Avellaneda gibt an, dass kein Interessenkonflikt besteht.

\section{Literatur}

1. Reilly LM, Ammar AD, Stoney RJ, Ehrenfeld WK (1985) Late results following operative repair for celiac artery compression syndrome. J Vasc Surg 2(1):79-91

2. Sultan S, Hynes N, Elsafty N, Tawfick W (2013) Eight years experience in the management of median arcuate ligament syndrome by decompression, celiac ganglion sympathectomy, and selective revascularization. Vasc Endovasc Surg 47(8):614-619. https://doi.org/10.1177/ 1538574413500536

3. Brown CL, Rizer M, Alexander R, Sharpe EE 3rd, Rochon PJ (2018) Pelvic congestion syndrome: systematic review of treatment success. Semin Intervent Radiol 35(1):35-40. https://doi.org/10 1055/s-0038-1636519

4. Ayloo SM, Masrur MA, Bianco FM, Giulianotti PC (2011) Robotic Roux-en-Y Duodenojejunostomy for Superior Mesenteric Artery Syndrome: Operative Technique. Journal of Laparoendoscopic \& Advanced Surgical Techniques 21(9):841-844. https://doi.org/10.1089/lap.2011.0070

5. Pourhassan S, Grotemeyer D, Fürst G, Rudolph J, Sandmann W (2008) Infrarenal transposition of the superior mesenteric artery: a new approach in the surgical therapy for Wilkie syndrome. J Vasc Surg 47(1):201-204.https://doi.org/10.1016/j.jvs.2007. 07.037

6. Shahi N, Arosemena M, Kwon J, Abai B, Salvatore D, DiMuzio P (2019) Functional popliteal artery entrapment syndrome: a review of diagnosis and management. Ann Vasc Surg. https://doi.org/10. 1016/j.avsg.2018.12.105 